\title{
Mass Spectrometric Detection and Fragmentation Patterns of Synthetically Useful Chromium and Tungsten Carbene Complexes
}

\author{
Torsten Weilandt, Ephraim Graeff, Jochen F. Schneider, Alexander W. Koch, Florian
}

A. Zschoche, Karl Heinz Dötz, and Christoph A. Schalley*

Kekulé-Institut für Organische Chemie und Biochemie der Universität, Gerhard-Domagk-Str.

1,D-53121 Bonn, Germany, email: c.schalley@uni-bonn.de

\section{Supporting Information}

\section{Selected mass spectra for other carbene complexes under study}

The following mass spectra have been selected to support those discussed in the main text (see references to supporting information there) and to provide some data for the tungsten carbene complexes which behave similarly as compared to the chromium analogues.

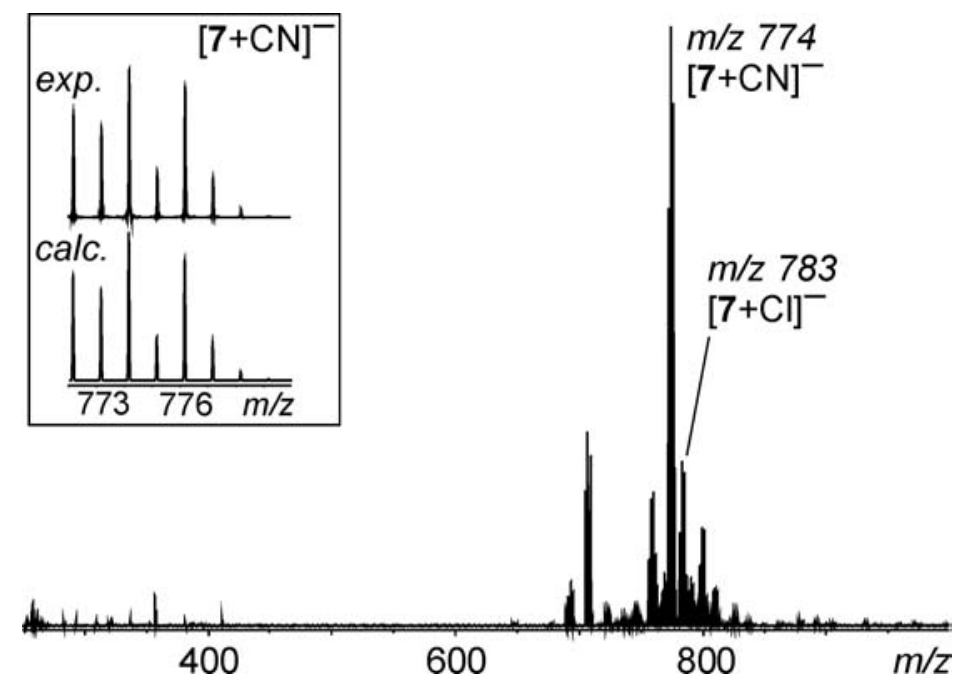

Figure S1. Negative ion ESI mass spectrum of the tungsten carbene complex 7 after addition of KCN. The inset compares the experimental isotope pattern to that calculated on the basis of natural abundances. 


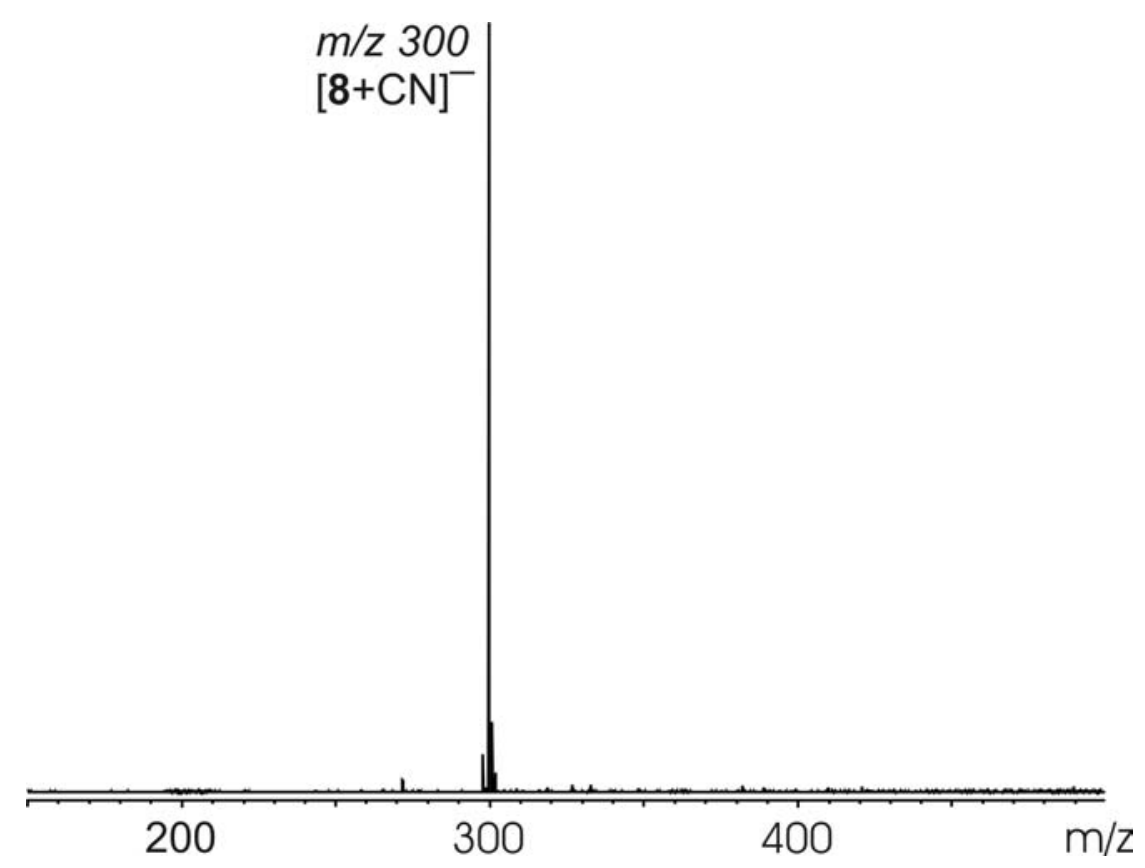

Figure S2. Negative ion ESI mass spectrum of the chromium carbene complex $\boldsymbol{8}$ after addition of $K C N$.
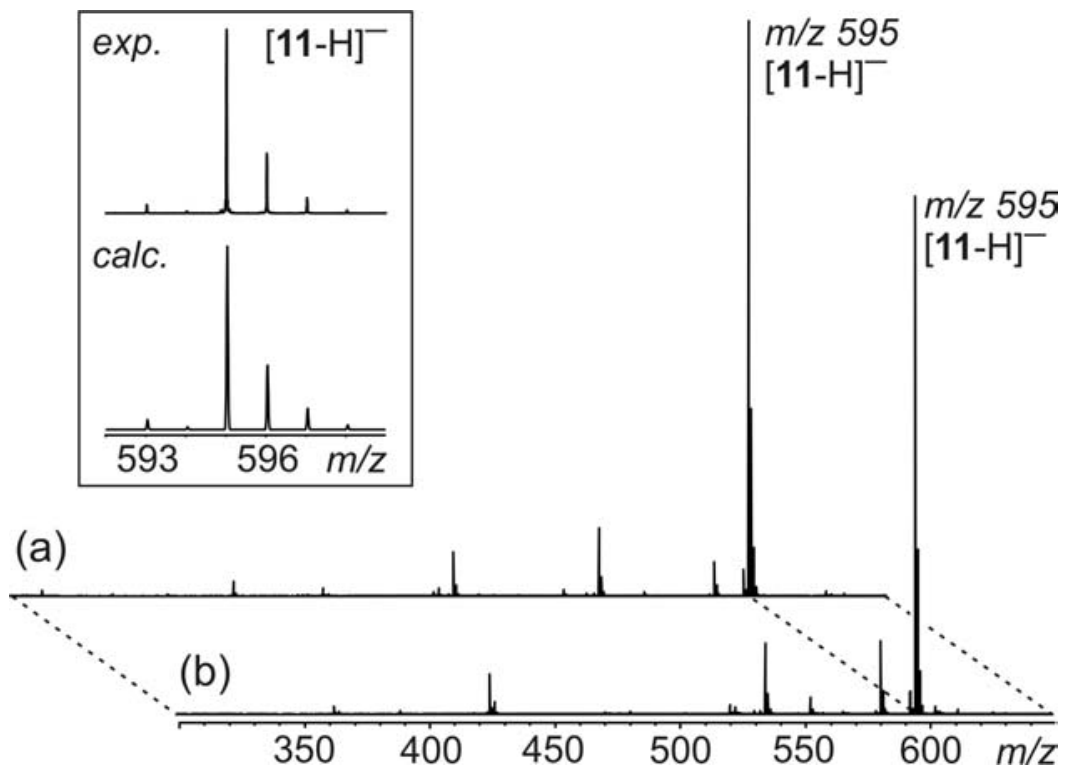

Figure S3. Negative ion ESI mass spectra of 11 sprayed from an acetonitrile solution (a) before and (b) after the addition of KCN. No cyanide adducts are observed. The inset compares the experimental isotope pattern with that calculated on the basis of natural abundances. 


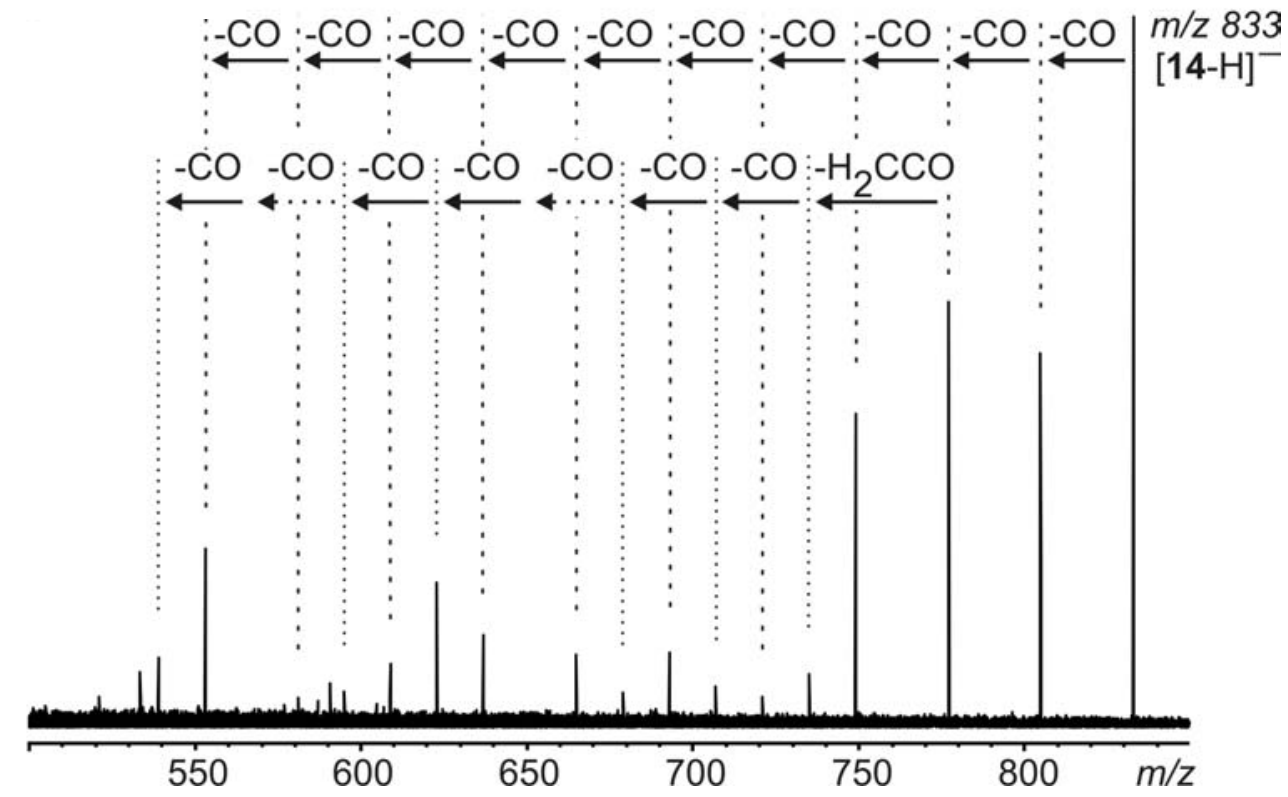

Figure S4. MS/MS spectra (CID) of mass-selected $[14-H]^{-}$. A second fragmentation channel competes with the $\mathrm{CO}$ losses and involves the formation of neutral ketene $\mathrm{CH}_{2}=\mathrm{C}=\mathrm{O}$.

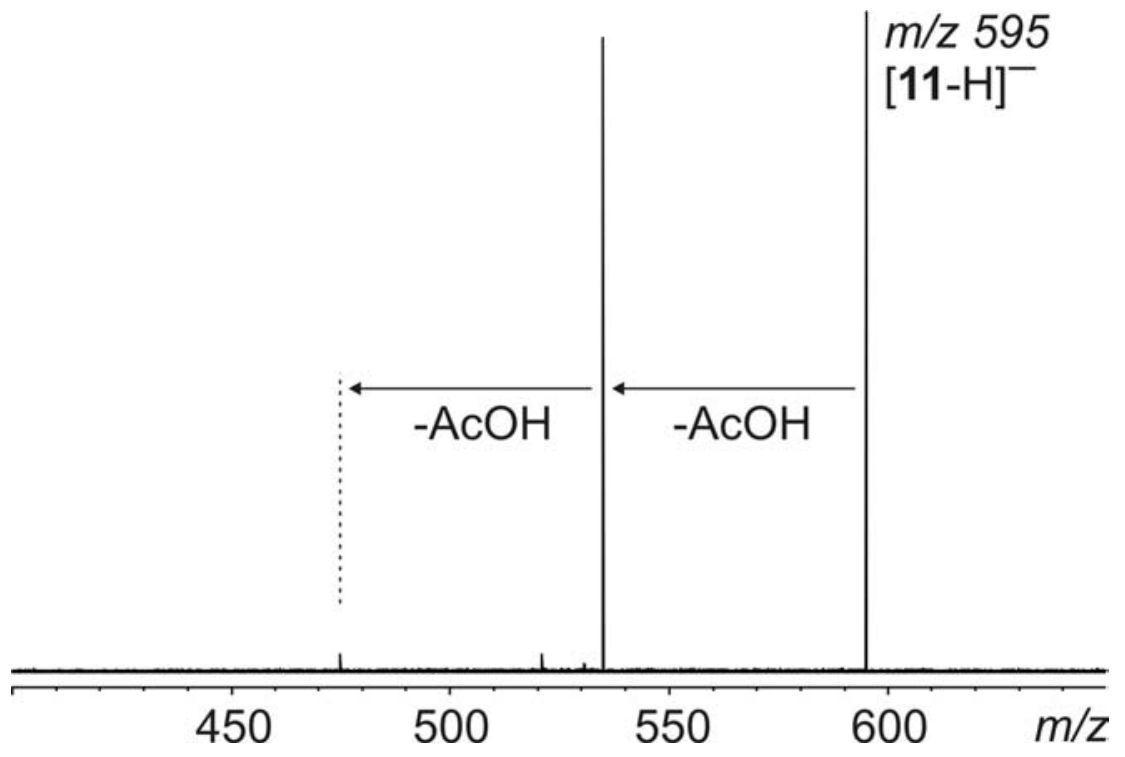

Figure S5. MS/MS spectrum (CID) of mass-selected [11-H] ${ }^{-}$. No carbon monoxide losses from the $\mathrm{Cr}(\mathrm{CO})_{5}$ moiety are observed. Instead 1,2-elimination reactions of acetic acid molecules occur from the sugar backbone. 


\section{Experimental Details}

Syntheses. All carbene complexes under study here were synthesized, purified, and characterized according to well-established literature procedures. ${ }^{1}$

Preparation of the samples. All sample solutions for ESI-MS analysis were prepared under inert gas conditions. The solvents used were dried and degassed by standard procedures.

ESI-FT-ICR mass spectrometry. ESI mass spectra were recorded on a Bruker APEX IV Fourier-transform ion-cyclotron-resonance (FT-ICR) mass spectrometer with an Apollo electrospray ion source equipped with an off-axis $70^{\circ}$ spray needle. Typically, acetonitrile or methanol served as the spray solvent and $30-50 \mu \mathrm{M}$ solutions of the analytes were used to which ca. 2 eq. of $\mathrm{KCN}$ were added in those cases where the cyanide complex of the chromium carbene complex was detected. Analyte solutions were introduced into the ion source with a syringe pump (Cole-Parmers Instruments, Series 74900) at flow rates of ca. 3 $4 \mu \mathrm{L} / \mathrm{min}$. Ion transfer into the first of three differential pumping stages in the ion source occurred through a glass capillary with $0.5 \mathrm{~mm}$ inner diameter and nickel coatings at both ends. Ionization parameters - some with a significant effect on signal intensities - were adjusted as follows: capillary voltage: +4.9 to $+4.2 \mathrm{kV}$; endplate voltage: +4.5 to $+3.7 \mathrm{kV}$; capexit voltage: -200 to $-20 \mathrm{~V}$; skimmer voltages: -5 to $-25 \mathrm{~V}$; temperature of drying gas:

\footnotetext{
${ }^{1}$ (a) Dötz, K. H.; Sturm, W.; Popall, M.; Riede, J. J. Organomet. Chem. 1984, 277, 267. (b) Dötz, K. H.; Neuß, O.; Nieger, M. Synlett, 1996, 995. (c) Tomuschat, P.; Nieger, M.; Dötz, K. H. Chem. Ber. 1997, 130, 1605. (d) Weyershausen, B.; Nieger, M.; Dötz, K. H. J. Org. Chem. 1999, 64, 4206. (e) Bühler, G.; Feiters, M. C.; Nolte, R. J. M.; Dötz, K. H. Angew. Chem. 2003, 115, 2599; Angew. Chem. Int. Ed. 2003, 42, 2494. (f) Gräff, E. Dissertation, Bonn, 2004. (g) Koch, A. W. Dissertation, Bonn, 2005. (h) Schneider, J. F.; Nieger, M.; Nättinen, K.; Dötz, K. H. Synthesis, in press. (i) Schneider, J. F.; Nieger, M.; Nättinen, K.; Lewall, B.; Niecke, E.; Dötz, K. H. Eur. J. Org. Chem., in press. (j) Gräff, E.; Nieger, M.; Dötz, K. H. Eur. J. Org. Chem., in preparation.
} 
$40{ }^{\circ} \mathrm{C}$. The flows of the drying and nebulizer gases were kept in a medium range (ca. 25 psi). The ions were accumulated in the instruments hexapole for $0.5-3 \mathrm{~s}$, introduced into the FTICR cell, which was operated at pressures below $10^{-10}$ mbar and detected by a standard excitation and detection sequence. For each measurement 8 to 128 scans were averaged to improve the signal-to-noise ratio.

Tandem MS experiments. For MS/MS experiments, the whole isotope patterns of the ions of interest were isolated by applying correlated sweeps, followed by shots to remove the higher isotopes. After isolation, argon was introduced into the ICR cell as the collision gas through a pulsed valve at a pressure of ca. $10^{-8}$ mbar. The ions were accelerated by a standard excitation protocol and detected after a $2 \mathrm{~s}$ pumping delay. A sequence of several different spectra was recorded at different excitation pulse attenuations in order to get at least a rough and qualitative idea of the effects of different collision energies on the fragmentation patterns. Instead of exciting the ions by collisions with argon, the Infrared Multiphoton Dissociation (IRMPD) laser can be used which operates at a tunable power of 0 to $25 \mathrm{~W}$ and can be adjusted with respect to the length of the irradiation time. For all compounds tested, the results of CID and IRMPD experiments was qualitatively identical so that we refrain from an in-depth discussion of the IRMPD experiments here. 